\title{
On interface dynamics
}

\author{
V. S. Arpaci and A. Esmaeeli \\ Department of Mechanical Engineering and Applied Mechanics, The University of Michigan, Ann Arbor, \\ Michigan 48109
}

(Received 24 September 1999; accepted 2 February 2000)

An intuitive study is presented for unstable interfacial waves. The maximum wavelength obtained for the most rapid unstable growth is shown to have a universal part which also characterizes the isotropic scales of buoyancy-driven turbulence. (c) 2000 American Institute of Physics. [S1070-6631(00)03005-1]

In the contemporary literature Rayleigh-Taylor instability continues to attract steady attention. The latest studies are directed toward nonlinear effects, chaos, and turbulence. ${ }^{1-3}$ An important conclusion emerging from these studies is that gravity $g$ and wavelength $\lambda$ are the governing basic scales from initial perturbations to turbulent mixing. The formation of smaller and smaller length scales is believed to be the result of repeated generations of Kelvin-Helmholtz instability ${ }^{4}$ which eventually leads to turbulence. The objective of this study is to support some of these conclusions, first by intuitive arguments based on linear instability theory, and second by the microscales of turbulence. ${ }^{5}$

Consider a viscous fluid of viscosity $\mu$ and density $\rho_{1}$ occupying the semi-infinite region $z<0$ and acted on by an effective driving buoyant force per unit mass, $b$. Let the semi-infinite region $z>0$ be occupied by a gas with density $\rho_{2}$ and negligible viscosity. Assume the interface to be disturbed by a plane wave of small amplitude $A_{k}(t) \exp (i k x), k$ being the wave number.

In terms of the well-known expression for a damped simple harmonic oscillator, the surface wave is expected to satisfy

$$
\frac{d^{2} A_{k}}{d t^{2}}+f(\nu, k) \frac{d A_{k}}{d t}+\omega^{2} A_{k}=0,
$$

where

$$
f(\nu, k) \sim \frac{1}{t} \sim \nu k^{2},
$$

$\nu$ and $t$ being the kinematic viscosity and time, respectively, and

$$
\omega(b, k) \sim \frac{1}{t} \sim(b k)^{(1 / 2)} .
$$

Then, with an unknown coefficient $C$, Eq. (1) can be written as

$$
\frac{d^{2} A_{k}}{d t^{2}}+C \nu k^{2} \frac{d A_{k}}{d t}+\omega^{2} A_{k}=0 .
$$

For small damping ${ }^{6}$ (long-wave limit),

$$
C=4, \quad \nu k^{2} \ll \omega^{2}
$$

and, for large damping ${ }^{7}$ (short-wave limit),

$$
C=2, \quad \nu k^{2} \gg \omega^{2} .
$$

We are interested in the unstable growth of waves in these limiting cases.

Now, let the semi-infinite region $z<0$ be occupied by the gas and the region $z>0$ by the viscous fluid, and assume $\omega^{2}=-\sigma^{2}$ for an unstable interface. The following intuitive arguments clarify the effective driving force in the case of buoyant flows. From a momentum balance,

$$
\rho V \sim F t,
$$

where $\rho \sim\left(\rho_{1}+\rho_{2}\right)$ is the effective density, $V$ a characteristic velocity, and

$$
F \sim g\left(\rho_{1}-\rho_{2}\right)-k^{2} S
$$

is the effective buoyant force per unit volume, $S$ being the surface tension. The sign of the surface tension term reflects its stabilizing effect. On dimensional grounds,

$$
t \sim \sigma^{-1}, \quad V \sim \sigma / k,
$$

and Eq. (5) gives

$$
\frac{\sigma}{k} \sim\left[\frac{g}{k}\left(\frac{\rho_{1}-\rho_{2}}{\rho_{1}+\rho_{2}}\right)-\frac{k S}{\rho_{1}+\rho_{2}}\right]^{1 / 2}=\left(\frac{b}{k}\right)^{1 / 2},
$$

where $b$ now includes surface tension,

$$
b=g A-\frac{k^{2} S}{\rho_{1}+\rho_{2}},
$$

and $A=\left(\rho_{1}-\rho_{2}\right) /\left(\rho_{1}+\rho_{2}\right)$ is the Atwood number.

For the case of short waves, Eq. (2) becomes

$$
\frac{d^{2} A_{k}}{d t^{2}}+2 \nu k^{2} \frac{d A_{k}}{d t}-\sigma^{2} A_{k}=0,
$$

which, with an amplitude growth, $A_{k}(t)=A_{k}(0) e^{n t}$, leads to

$$
n^{2}+2 \nu k^{2} n-b k=0 \text {. }
$$

Then, $d n / d k=0$ gives the wavelength of the interface which corresponds to the most rapid growth of the short waves for $\lambda_{\max }=4 \pi\left(\nu^{2} / b\right)^{1 / 3}$, where $\lambda k=2 \pi$. Similar steps for the case of long waves lead to $\lambda_{\max }=4 \pi\left(4 \nu^{2} / b\right)^{1 / 3}$ and demonstrate the fact that the wavelength of the most rapid growth of any interface wave is 


$$
\lambda_{\max }=C\left(\frac{\nu^{2}}{b}\right)^{1 / 3},
$$

where $\lambda_{\max }$ for both waves turns out to have a universal part, $\left(\nu^{2} / b\right)^{1 / 3}$, and a structural part, $C$, which is a numerical constant depending on the size of the wave. We now demonstrate this fact by other intuitive arguments based on the microscales of turbulence which characterize the limit of the cascade of smaller waves.

Let the instantaneous interface velocity of a buoyancydriven turbulent flow be decomposed into temporal means and fluctuations, $U_{i}+u_{i}, U_{i}$ being the mean velocity oscillations perturbed by the turbulent root-mean-square fluctuations, $u_{i}$. Then, the balance of the mean kinetic energy of the homogeneous rms velocity fluctuations is

$$
\mathcal{B} \sim \epsilon \sim \mathcal{P}
$$

where $\mathcal{B}=w b$ is the effective energy production by buoyant forces, $w$ being the rms of turbulent velocity fluctuations (or turbulent intensity), $\epsilon=2 \nu \overline{s_{i j} s_{i j}}$ is the dissipation of turbulent kinetic energy, $s_{i j}$ being the rate of fluctuating strain, and $\mathcal{P}=-\overline{u_{i} u_{j}} S_{i j}$ is the inertial production of turbulent kinetic energy, $S_{i j}$ being the rate of mean strain.

On dimensional grounds, Eq. (9) gives

$$
\mathcal{B} \sim \frac{\nu w^{2}}{\delta^{2}} \sim \frac{w^{3}}{h},
$$

where $\delta$ denotes a dissipation length scale and $h$ a production length scale for homogeneous turbulence.

The velocity obtained from Eq. (10) in terms of $\mathcal{B}$,

$$
w \sim \delta\left(\frac{\mathcal{B}}{\nu}\right)^{1 / 2} \sim(\mathcal{B} h)^{1 / 3},
$$

leads for $\delta$ to

$$
\delta \sim h^{1 / 3}\left(\frac{\nu^{3}}{\mathcal{B}}\right)^{1 / 6} .
$$

For the isotropic turbulence, repeating the foregoing developments in terms of one scale alone, that is, replacing $\delta$ and $h$ with $\eta$ in Eq. (12), we obtain a Kolmogorov scale,

$$
\eta \sim\left(\frac{\nu^{3}}{\mathcal{B}}\right)^{1 / 4}
$$

Now, in terms of the isotropic velocity $w \sim \nu / \eta$, obtained from Eq. (10) by replacing $\delta$ and $h$ with $\eta$, the energy input becomes

$$
\mathcal{B}=b w \sim b\left(\frac{\nu}{\eta}\right) .
$$

Expressing $\mathcal{B}$ of Eq. (13) in terms of Eq. (14) yields

$$
\eta \sim\left(\frac{\nu^{2}}{b}\right)^{1 / 3},
$$

which is identical in form to Eq. (8).

The foregoing dimensional arguments involving linear instability theory and the microscales of turbulence show that the viscous (dissipative) aspects of interface dynamics lead to a scale, $C\left(\nu^{2} / b\right)^{1 / 3}$, which has a universal part, $\left(\nu^{2} / b\right)^{1 / 3}$, independent of isotropic flow conditions. Only the numerical constant, $C$, related to this scale varies depending on the flow structure. This constant for the short waves is known to be $4 \pi$, for the long waves to be $4^{1 / 3}(4 \pi)$, and, for the Kolmogorov scale, is usually assumed ${ }^{8}$ to be on the order of one. Accordingly, for the same buoyant force, the wavelength of the Kolmogorov waves is expected to be an order of magnitude smaller than the wavelength of the RayleighTaylor waves.

Note that the contemporary experimental and computational studies so far have been mostly devoted to large (production) scales of turbulence which are a measure of the structural evolution of interface dynamics. ${ }^{1-3,9-13}$ Here, we obtain the well-known mixing scale by an inertial estimate for turbulent intensity. From Eq. $(11), w \sim(\mathcal{B} h)^{1 / 3}$, in terms of $b=\mathcal{B} / w$, we have $w \sim(b h)^{1 / 2}$, which, in view of $w$ $\sim h / t$, becomes $h \sim b t^{2}$, or, explicitly,

$$
h=\alpha\left[g A-\frac{k^{2} S}{\rho_{1}+\rho_{2}}\right] t^{2},
$$

where $k$ is a measure of curvature and scales with the Taylor scale. From Eq. (10), $\delta^{2} \sim \nu h / w$, in terms of $w \sim h / t$, we get $\delta^{2} \sim \nu t$, which in view of $k \sim \delta^{-1}$ gives $k^{2} \sim(\nu t)^{-1}$. Accordingly,

$$
h=\alpha\left[g A-\frac{S / \nu t}{\rho_{1}+\rho_{2}}\right] t^{2} .
$$

In the absence of surface tension, Eq. (16) reduces to

$$
h=\alpha g A t^{2} \text {. }
$$

This scale is known to characterize the highly disordered mixing zone which follows the nonlinear growth of interface. $^{3,9-13}$ The experimental studies appear to support this relation for the behavior of light fluid (bubbles). Also the ratio of spike penetration to bubble penetration is known to be a weak function of density ratio, $h_{2} / h_{1}=f\left(\rho_{1} / \rho_{2}\right)$, which may equivalently be written as $h_{2} / h_{1}=f(A)$. Furthermore, $h_{2} / h_{1}=1$ for the Boussinesq limit $\left(\rho_{1} / \rho_{2} \sim 1\right)$ suggests

$$
\frac{h_{2}}{h_{1}}=1+f(A) .
$$

However, for a better understanding of spike and bubble penetrations, more experiments involving systematic parametric variations with improved diagnostics are needed. The recent study by Dimonte and Schneider ${ }^{9}$ further supports this need.

\section{ACKNOWLEDGMENTS}

This work was supported by NASA Microgravity under Contract No. NAG3-1952 96-3063 with Dr. David Chao as technical monitor.

${ }^{1}$ X. He, R. Zhang, S. Chen, and G. Doolen, "'On the three-dimensional Rayleigh-Taylor instability,', Phys. Fluids 11, 1143 (1999).

${ }^{2}$ A. Oparin and S. Abarzhi, "Three-dimensional bubbles in RayleighTaylor instability,', Phys. Fluids 11, 3306 (1999).

${ }^{3}$ S. N. Dalziel, P. F. Linden, and D. L. Youngs, 'Self-similarity and internal structure of turbulence induced by Rayleigh-Taylor instability, " J. Fluid Mech. 399, 1 (1999). 
${ }^{4}$ D. H. Sharp, “An overview of Rayleigh-Taylor instability," Physica D 12, 3 (1984).

${ }^{5}$ V. S. Arpaci, Microscales of Turbulence (Gordon and Breach, New York, 1997).

${ }^{6}$ M. S. Plesset and C. G. Whipple, "Viscous effects in Rayleigh-Taylor instability," Phys. Fluids 17, 1 (1974).

${ }^{7}$ H. Lamb, Hydrodynamics, 6th ed. (Dover, New York, 1945).

${ }^{8}$ H. Tennekes and J. L. Lumley, A First Course in Turbulence (MIT, Cambridge, 1972).

${ }^{9}$ G. Dimonte and M. B. Schneider, "Density ratio dependence of
Rayleigh-Taylor mixing for sustained and impulsive acceleration histories," Phys. Fluids 12, 304 (2000).

${ }^{10}$ M. B. Schneider, G. Dimonte, and B. Remingtone, "Large and small scale structure in Rayleigh-Taylor mixing,' Phys. Rev. Lett. 80, 3507 (1998).

${ }^{11}$ D. L. Youngs, "Numerical simulation of mixing by Rayleigh-Taylor and Richtmyer-Meshkov instabilities," Laser Part. Beams 12, 725 (1994).

${ }^{12} \mathrm{D}$. L. Youngs, "Three-dimensional numerical simulation of turbulent mixing by Rayleigh-Taylor instability," Phys. Fluids A 3, 1312 (1991).

${ }^{13}$ D. L. Youngs, "Modeling turbulent mixing by Rayleigh-Taylor instability," Physica D 37, 270 (1989). 